\title{
Biocompatibility of a chlorhexidine local delivery system in a subcutaneous mouse model
}

\author{
Adriana-Socorro-Ferreira Monteiro ${ }^{1}$, Luís-Guilherme-Scavone Macedo ${ }^{2}$, Nelson-Luiz Macedo ${ }^{3}$, Fernanda- \\ Alves Feitosa ${ }^{4}$, Thiago Toyoshima ${ }^{5}$
}

\author{
${ }^{1}$ DDS, MSc, PhD, Bioscience Center for Special Health Care Needs -CEBAPE/UNESP - São Paulo State University - São José \\ dos Campos Dental School \\ ${ }^{2}$ DDS, MSc, Pos-graduate, Restorative Dentistry, Department of Dental Materials and Prosthesis - UNESP - São Paulo State \\ University - São José dos Campos Dental School \\ ${ }^{3}$ DDS, MSc, PhD, Assistant Professor, Department of Diagnosis and Surgery, Periodontics Division - UNESP - São Paulo State \\ University - São José dos Campos Dental School \\ ${ }^{4}$ Graduate student - UNESP - São Paulo State University - São José dos Campos Dental School \\ ${ }^{5}$ DDS, Department of Diagnosis and Surgery, Periodontics Division - UNESP - São Paulo State University - São José dos Cam- \\ pos Dental School
}

Correspondence:

Department of Diagnosis and Surgery,

São José dos Campos Dental School, UNESP

Avenida Engenheiro Francisco José Longo, 777

Caixa Postal 314 CEP 12245-000

São José dos Campos, SP - Brazil

driffmonteiro@yahoo.com.br

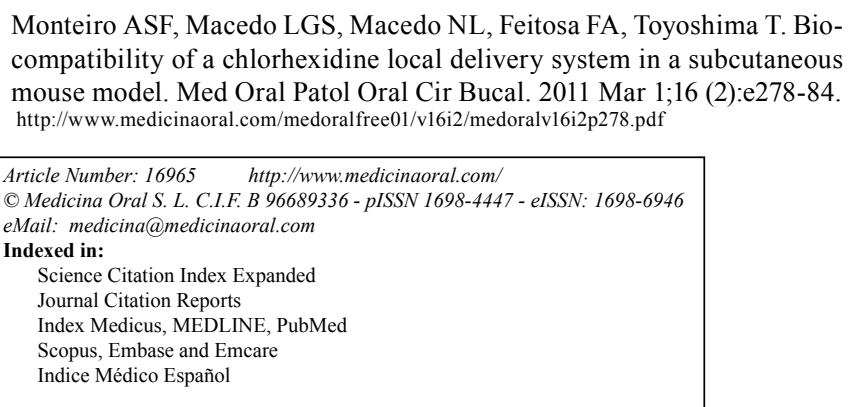

\begin{abstract}
Objective: This study aimed evaluating histologically and histomorphometrically the response of the conjunctive tissue face to the implant of chlorhexidine chips in the subcutaneous tissues of rats. Study Design: In this research 35 male rats Wistar were used to analyze the biocompatibility and the degradation process of chlorhexidine chip. In each animal, it was made 2 incisions for subcutaneous implantation of chlorhexidine chip (test group) and a polytetrafluorethylene membrane (control group). The morphological changes in subcutaneous implantations were assessed after 1, 3, 5, 7, 10, 14, 21 days. The data were submitted to Friedman nonparametric test to analyze the comparisons among observation periods and to allow the comparison among groups. Results: Differences were found in the analysis of the inflammatory response when comparing the tested materials ( $p$ values $\leq 0.05$ ). In test group was observed hemorrhage, edema and intense inflammatory infiltrate predominantly neutrophilic around material. From 3-day and subsequent periods was verified granulation tissue externally at this infiltrate. From 10-day on was observed crescent area of degradation of chlorhexidine chip, associated with neutrophilic and macrophagic infiltrate, that maintained until 21-day. In the control group, moderate inflammatory infiltrate was observed initially, predominantly polymorphonuclear, edema and granulation tissue 3-day period. The inflammatory infiltrate was gradually replaced for granulation tissue, culminating in a fibrous capsule. Giant multinucleate cells situated at contact interface with the coating was examined since 3-day and persisted until 21-day. Conclusion: The chlorhexidine chip induces an intense acute inflammatory response at subcutaneous tissue of rats. Therefore, at conditions of this study was not biocompatible.
\end{abstract}

Key words: Biocompatibility, chlorhexidine toxicity, drug delivery system, periodontitis. 


\section{Introduction}

Periodontal disease represents a variety of clinical manifestations of a heterogeneous group of infectious disorders. Periodontitis is an episodic and segmental inflammatory infection and the natural history of the infection is highly variable. Some patients remain stable for many years, while other patients have a history of sporadic or gradually progressing disease (1).

The vast majority of periodontitis cases responded well to conventional nonsurgical periodontal therapy, i.e. scaling and root planning (SRP), improved oral hygiene and supportive recall. However, certain patients, for various reasons, do not respond favorably to mechanical therapy alone. For these patients, the use of an appropriate adjunctive antimicrobial is often beneficial (2).

Current periodontal therapy strongly emphasizes suppressing or eradicating specific pathogens. However, mechanical debridement may fail to remove pathogenic organisms because of their location in subepithelial gingival tissue, crevicular epithelial cells, collagenous substrata, altered cementum and radicular dentinal tubuli, subgingival hard deposits, furcations or other anatomic features complicating adequate instrumentation. Moreover, pathogens frequently colonize oral mucosa, tongue dorsum, tonsils and other buccal domains and may translocate from non-periodontal sites to periodontal crevices (3-4).

As an antiseptic, chlorhexidine (CHX) has been used effectively for over than 30 years in the treatment of gingival inflammation. It shows a broad spectrum of topical antimicrobial activity, effectiveness and substantivity. On the other hand, subgingival irrigation using CHX was not effective in the treatment of periodontitis because of the lack of significant concentrations for sufficient lengths of time within the periodontal pocket (3).

Local administration, therefore, may be considered an alternative to overcome these problems. Improved patient compliance, drug access to the site of disease, and lower total drug dosage are the main benefits of a controlled delivery device (5).

A degradable, subgingivally placed drug delivery system containing $2.5 \mathrm{mg}$ chlorhexidine gluconatet was developed(6), but experimental and clinical studies demonstrated controversial results about the use of CHX chip as an adjunct of SRP in the treatment of periodontal disease (3-10).

A strong anti-inflammatory effect was associated to $\mathrm{CHX}$, by reducing the activity of matrix metalloproteinases (MMPs) (7), the basal concentration of leucocytes and the levels of the proinflammatory cytokines (11). However, it has been related toxicity effects from CHX (12-15), even at a very low concentration (16). This drug has been demonstrated to induce an inflammatory reaction (14), tissue necrosis (15) and to retard granulation tissue formation and wound healing (13). Besides, CHX behaved in a cytotoxic manner toward osteoblasts in vitro (17).

Based on the exposed, the aim of this experimental study was to evaluate histologically and histomorphometrically the response of the conjunctive tissue face to the implant of CHX chips in the subcutaneous tissues of rats. The null hypothesis was that there would be no difference between the results obtained after CHX chips implantation in rats subcutaneous. The alternative hypothesis was that there would be a statistically significant difference.

\section{Materials and Methods}

-Microscopic analysis

The biocompatibility of two types of materials was evaluated by morphological alterations in subcutaneous tissue of 35 male Wistar rats, weighting between 150 and $200 \mathrm{~g}$. This research was approved by the Committee of Ethics in Animal Research of São José dos Campos School of Dentistry - UNESP, according to the criteria of the animal protocol.

The chlorhexidine-gelatin is a bioresorbable chip containing $2.5 \mathrm{mg}$ of chlorhexidine in a gelatin matrix. The chip is approximately $5 \mathrm{~mm}$ height and was developed to be placed in an isolated periodontal pocket. In its present formulation, the chip resorbs and releases chlorhexidine in the pocket for approximately 7 to 10 days following placement. The average CHX concentration remain above $150 \mathrm{ppm}$ at least 7 days (18).

Polytetrafluoroethylene (PTFE) nonporous membrane (Tecnoflon-Brasflon, Ind. \& Com. Plasticos, São Paulo, $\mathrm{SP}$ ), with $0.13 \mathrm{~mm}$ thickness, presents characteristics and biocompatibility appropriate to be applied as an inflammatory reaction standard material of comparison with the material tests(19-20).

Preoperatively, the animals were weighed and sedated with analgesic-sedative and muscle-relaxing $(0.1 \mathrm{~mL} / 100 \mathrm{~g})$ Rompum $(2 \%$ aqueous solution of $2-(2,6-$ xilidine)-5,6-dihydro-4H-1,3 thiazin, Bayer, Brazil) five minutes before administration of general anesthesia $(0.5 \mathrm{~mL} / 100 \mathrm{~g})$ Dopalen (ketamine hydrochloride, Agribrands do Brasil Ltda, Brazil).

After trichotomy and the asepsis of the dorsal region, two longitudinal incisions ( $2 \mathrm{~cm}$ extension) at a distance of $4 \mathrm{~cm}$ were performed in the medium line. The blunt dissection in the subcutaneous connective tissue was made through laterally with a round-tip scissor to material placement. The membrane was cut in disks of $1 \mathrm{~cm}$ diameter, and later implanted in the subcutaneous tissue. The CHX chip (test group) was inserted on the right side and the PTFE (control group) was placed on the left side. The surgical wound was closed with 4-0 mononylon-interrupted sutures, for a first intention repair. The animals were euthanized at 1, 3, 5, 7, 10, 14, 21 days 
with an anesthetic overdose. The samples containing the material were immediately fixed in $10 \%$ formalin solution and routinely processed for histology. Longitudinal histological sections with $6 \mu \mathrm{m}$ thickness were stained with hematoxylin and eosin for analysis in light microscopy.

\section{-Histomorphometric analysis}

The central point of histological section randomization and selection for histomorphometric analysis was accomplished randomly, eliminating the occurrence of sampling bias(20). The chosen tissue area was submitted for examination with serial microscopic sections. From these sections, 3 were randomly chosen for histomorphometric analysis. Subsequently, 8 histological fields from each section, in the surgical region, were analyzed. At this step, a 40X objective (A-Plan, Carl Zeiss, Gottingen, Germany) and an ocular 10X (W-PI, Carl Zeiss, Gottingen, Germany) of an optical microscope (Axioskop 40, Carl Zeiss, Gottingen, Germany) were used.

Histomorphometric analysis was performed to find out qualitative parameters of the inflammatory reaction (19). A blind study was conducted to measure the tissue outcome in the area using scores, observing the presence of neutrophils, lymphocytes, plasmocytes, eosinophils, macrophages. Based on these findings, the slides were then classified according to the following scores: 0 - Absence (when inflammatory cells were absent or found only within the blood vessels); 1 - Dis- crete (when inflammatory cells were sparsely present or in very small groups); 2 - Moderate (when inflammatory cells were densely present or in some groups); 3 - Intense (when inflammatory cells were found in the entire field or were present in a large number, configuring great severity).

After qualitative analysis and attribution of scores to the observed events, the slides were scored according to inflammation severity. A ranking scale was established, which ordered the slides in increasing degree of inflammatory reaction, ranging from absent to high reaction.

The data were submitted to Friedman nonparametric test (Bioestat 5.0 for Windows; Belém, Pará, Brazil) to analyze the comparisons among observation periods and to allow the comparison among groups (5\% significance).

\section{Results}

Control group: The histological analysis showed a moderated initial inflammatory infiltrate with polymorphonuclear predominance, edema and granulation tissue. The inflammatory infiltration was gradually substituted by granulation tissue, which then turned into a fibrous capsule formation. Some multinucleated giant cells presented in the contact interface were observed since the 3-day and persisted until the 21-day (Fig. 1).

Test group: The histological analysis in the 1-day period showed hemorrhage, edema and an intense inflammatory infiltrate with predominance of polymorphonuclear

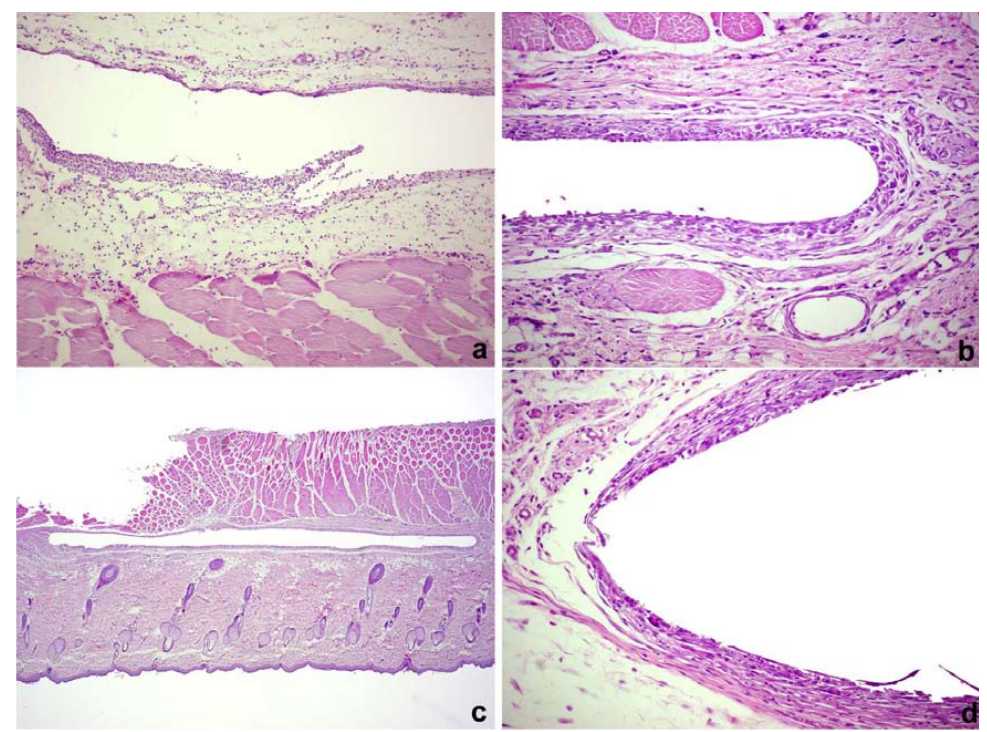

Fig. 1. Histological images of the PTFE group: a) 1 day: moderate inflammatory infiltrate around the material (Hematoxylin and eosin stain - 100x); b) 7 days: granulation tissue and local inflammatory infiltrate (Hematoxylin and eosin stain - 200x); c) 10 days: panoramic view of the place previously occupied by the membrane (Hematoxylin and eosin stain $-25 x)$; d) 21 days: fibrous capsule with some giant cells (Hematoxylin and eosin stain -200x). 


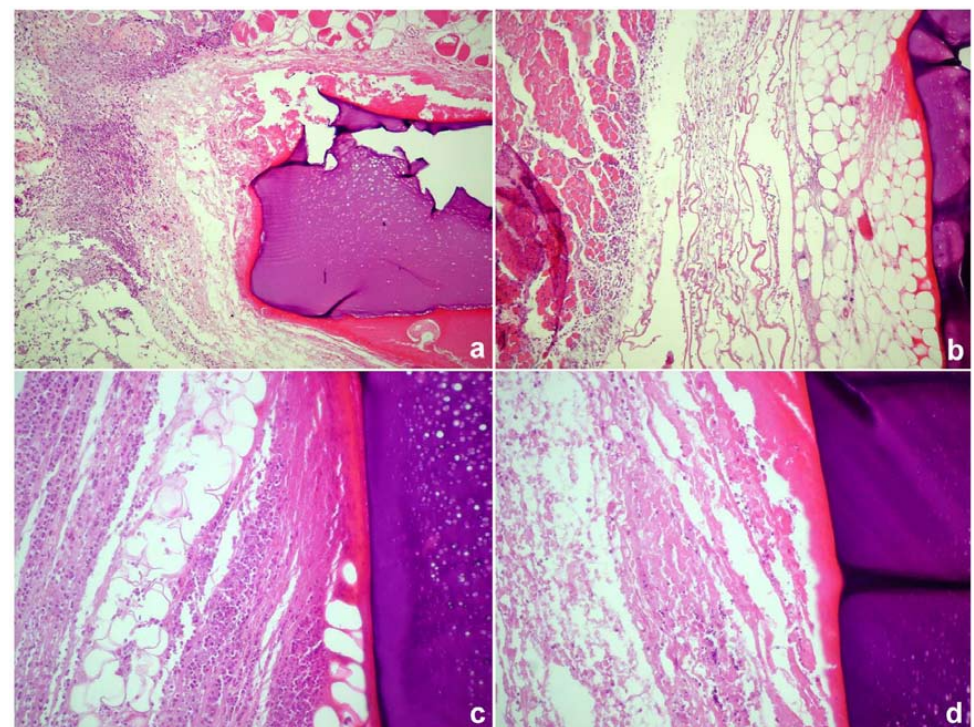

Fig. 2. Histological images of the CHX group: a) 1 day: intense and diffuse inflammatory infiltrate, edema and fibrin net around the material (Hematoxylin and eosin stain $-100 x)$; b) 3 days: edema and fibrin net around the material. Inflammatory infiltrate permeating the muscle fibers (Hematoxylin and eosin stain - 100x); c) 5 days: intense and difuse inflammatory infiltrate and edema around the material (Hematoxylin and eosin stain $-200 x)$; d) 7 days: edema and necrotics area close to the material (Hematoxylin and eosin stain - 200x).

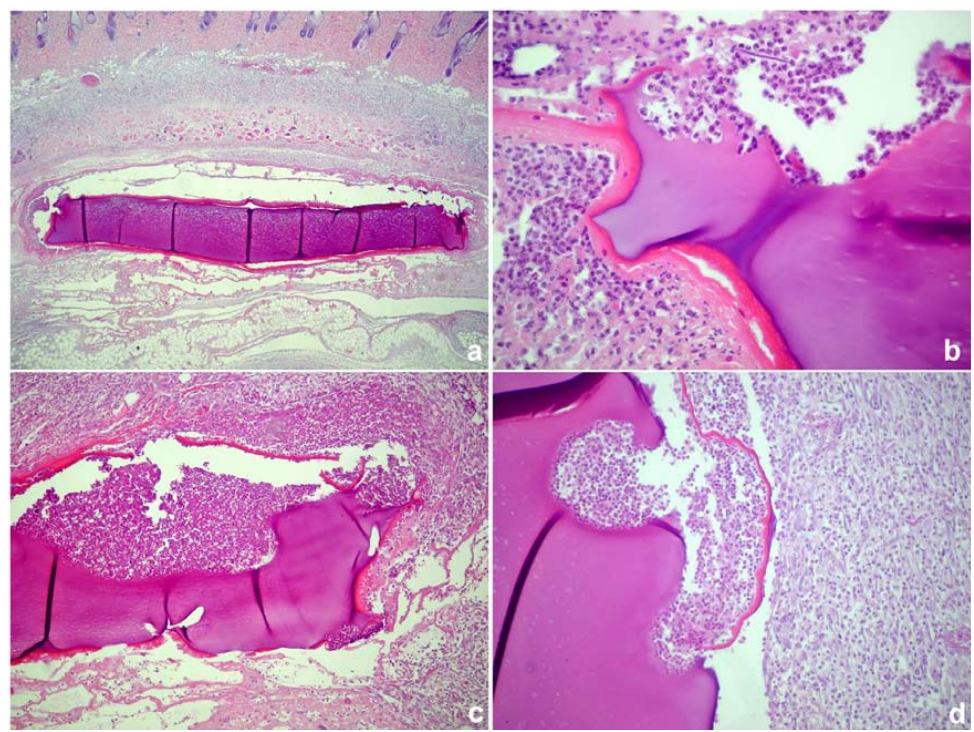

Fig. 3. Histological images of the CHX group: a) 10 days: panoramic view of the degradation lacuna of CHX chip (Hematoxylin and eosin stain - 25x); b) 10 days: degradation lacunas filled by neutrophils and macrophages (Hematoxylin and eosin stain - 400x); c) 14 days: degradation lacunas (Hematoxylin and eosin stain - 100x); d) 21 days: macrophages and neutrophils in the degradation lacuna. Externally, granulation tissue with mononuclear infiltrated (Hematoxylin and eosin stain $-200 x$ ). 
Table 1. Mean values and standard deviation of the inflammatory reaction histomorphometry of the studied group.

\begin{tabular}{|c|c|c|c|c|c|c|c|c|c|c|}
\hline & \multicolumn{2}{|c|}{ Sum of ranks } & \multicolumn{2}{|l|}{ Median } & \multicolumn{2}{|c|}{ Average rank } & \multicolumn{2}{|c|}{ Average values } & \multicolumn{2}{|c|}{ Standard deviation } \\
\hline & $\begin{array}{l}\mathrm{CHX} \\
4,320,00\end{array}$ & $\begin{array}{l}\text { PTFE } \\
2,315,00\end{array}$ & $\mathrm{CHX}$ & PTFE & $\mathrm{CHX}$ & PTFE & $\mathrm{CHX}$ & PTFE & $\mathrm{CHX}$ & PTFE \\
\hline 1 day & $\begin{array}{r}0 \\
4,125,00\end{array}$ & $\begin{array}{r}0 \\
2,250,00\end{array}$ & 30,000 & 20,000 & 108,000 & 57,875 & 29,750 & 22,000 & 0,1581 & 0,4051 \\
\hline 3 days & $\begin{array}{r}0 \\
4,120,00\end{array}$ & $\begin{array}{r}0 \\
2,315,00\end{array}$ & 30,000 & 20,000 & 103,125 & 56,250 & 29,000 & 21,750 & 0,3038 & 0,3848 \\
\hline 5 days & $\begin{array}{r}0 \\
4,110,00\end{array}$ & $\begin{array}{r}0 \\
2,245,00\end{array}$ & 30,000 & 20,000 & 103,000 & 57,875 & 29,000 & 22,000 & 0,3038 & 0,4051 \\
\hline 7 days & $\begin{array}{r}0 \\
4,075,00\end{array}$ & $\begin{array}{r}0 \\
2,000,00\end{array}$ & 30,000 & 20,000 & 102,750 & 56,125 & 29,000 & 21,750 & 0,3038 & 0,3848 \\
\hline 10 days & $\begin{array}{r}0 \\
3,940,00\end{array}$ & $\begin{array}{r}0 \\
1,455,00\end{array}$ & 30,000 & 20,000 & 101,875 & 50,000 & 28,750 & 20,750 & 0,3349 & 0,3499 \\
\hline 14 days & $\begin{array}{r}0 \\
3,855,00\end{array}$ & 0 & 30,000 & 20,000 & 98,500 & 36,375 & 28,250 & 17,750 & 0,3848 & 0,5305 \\
\hline $\begin{array}{l}21 \text { days } \\
\text { Friedman }(\mathrm{Fr})\end{array}$ & $\begin{array}{r}0 \\
2,605,48 \\
6\end{array}$ & 875,000 & 30,000 & 10,000 & 96,375 & 21,875 & 28,000 & 14,000 & 0,4051 & 0,4961 \\
\hline degrees of freedom & 13 & & & & & & & & & \\
\hline (p) & $<0.0001$ & & & & & & & & & \\
\hline
\end{tabular}

neutrophils adjacent to the material. The 3-day and subsequent periods showed a granulate tissue outside this infiltrate. In the 10-day period, degradation of the material was detected, mainly characterized by degradation lacunas filled by neutrophils and macrophages. The degradation process increased with time and persisted until the 21-day period (Figs. 2, 3).

\section{-Histomorphometric analysis}

The mean values and standard deviation of the inflammatory reaction histomorphometry of the studied groups for the different periods are shown in table 1 . Differences were found in the analysis of the inflammatory response when comparing the tested materials ( $p$ values were $\leq 0.05$ ). Nevertheless, when testing the influence of the time on the degree of inflammation, the inflammatory process did not decrease in a longer experimental interval in the CHX group. In assessing the difference between groups, there was a statistically significant difference in the evolution of the inflammatory reaction $(\mathrm{p}<0.0001)$. When analyzing the evolution of the inflammatory response over time, there were differences in the PTFE group, while there was no difference over time in CHX group (Table 2).

\section{Discussion}

The result of this study indicated that CHX chip behaved in a cytotoxic manner toward subcutaneous tissues of rats. A strong reaction was detected, in the histological analysis, characterized by hemorrhage, edema and an intense inflammatory infiltrate, predominantly neutrophilic, adjacent to the material. In the subsequent periods was verified granulation tissue externally at this infiltrate and crescent area of degradation of CHX chip, associated with neutrophilic and macrophagic cells.
However, according to some studies $(5,7,11)$, CHX can modulate an anti-inflammatory response. Houri-Haddad et al. (11) showed a decrease of the basal concentration of leucocytes and the levels of the proinflammatory cytokines using CHX diluted to $2000 \mathrm{mg} / \mathrm{ml}$ in phosphate-buffered saline. CHX chip may also reduce the level of prostaglandin E2, a proinflammatory agent that can induce pathologic tissue alterations, on gingival crevicular fluid (5). By immunofluorometric assay (IFMA) (7), it was observed a decrease in the activity of matrix metalloproteinases (MMPs), an important collagenases in the development of the inflammatory process (21). An inflammatory reaction induced by $\mathrm{CHX}$ has been demonstrated (13-14, 16-17, 22). Chang et al. (12) confirmed the toxicity of CHX on cultured human periodontal ligament by inhibiting double-stranded nucleic acid content, protein synthesis, and mitochondrial activity. The present research is in agreement with these studies that found an intense acute inflammatory response in the test group. Even in a low concentration, CHX have serious toxic effects on gingival fibroblasts and may affect wound healing (16). In an in vitro study, Giannelli et al. (22) suggested that the direct application of CHX during regenerative therapy for the treatment of periodontal disease could have serious toxic effects on gingival fibroblasts, endothelial cells and, specially, on alveolar osteoblasts, thus negatively interfering with the early healing phase of the oral disease. CHX may induce cell death by apoptosis and necrosis in cultured L929 fibroblasts via endoplasmic reticulum stress (13) and have some in vitro cytotoxic effect on osteoblasts (17).

Clinical studies evaluating the use of CHX chip in the treatment of periodontal disease presents promising results. A multicenter clinical trial (6) indicated that the 
Table 2. Friedman test of the inflammatory reaction histomorphometry of the studied group.

\begin{tabular}{|c|c|c|c|c|c|}
\hline Comparisons & Difference & $(p)$ & Comparisons & Difference & $(p)$ \\
\hline Rank $\mathrm{CHX} 1$ e $\mathrm{CHX} 3$ & 19,5 & ns & Rank CHX 10 e $\mathrm{CHX} 14$ & 13,5 & ns \\
\hline Rank CHX 1 e CHX 5 & 20 & ns & Rank CHX 10 e CHX 21 & 22 & ns \\
\hline Rank CHX 1 e $\mathrm{CHX} 7$ & 21 & ns & Rank CHX 10 e PTFE 1 & 176 & $<0.05$ \\
\hline Rank CHX 1 e $\mathrm{CHX} 10$ & 24,5 & ns & Rank CHX 10 e PTFE 3 & 182,5 & $<0.05$ \\
\hline Rank CHX 1 e CHX 14 & 38 & ns & Rank CHX 10 e PTFE 5 & 176 & $<0.05$ \\
\hline Rank CHX 1 e $\mathrm{CHX} 21$ & 46,5 & ns & Rank CHX 10 e PTFE 7 & 183 & $<0.05$ \\
\hline Rank CHX 1 e PTFE 1 & 200,5 & $<0.05$ & Rank CHX 10 e PTFE 10 & 207,5 & $<0.05$ \\
\hline Rank CHX 1 e PTFE 3 & 207 & $<0.05$ & Rank CHX 10 e PTFE 14 & 262 & $<0.05$ \\
\hline Rank CHX 1 e PTFE 5 & 200,5 & $<0.05$ & Rank CHX 10 e PTFE 21 & 320 & $<0.05$ \\
\hline Rank CHX 1 e PTFE 7 & 207,5 & $<0.05$ & Rank CHX 14 e CHX 21 & 8,5 & ns \\
\hline Rank CHX 1 e PTFE 10 & 232 & $<0.05$ & Rank CHX 14 e PTFE 1 & 162,5 & $<0.05$ \\
\hline Rank CHX 1 e PTFE 14 & 286,5 & $<0.05$ & Rank CHX 14 e PTFE 3 & 169 & $<0.05$ \\
\hline Rank CHX 1 e PTFE 21 & 344,5 & $<0.05$ & Rank CHX 14 e PTFE 5 & 162,5 & $<0.05$ \\
\hline Rank $\mathrm{CHX} 3$ e $\mathrm{CHX} 5$ & 0,5 & ns & Rank CHX 14 e PTFE 7 & 169,5 & $<0.05$ \\
\hline Rank CHX 3 e $\mathrm{CHX} 7$ & 1,5 & ns & Rank CHX 14 e PTFE 10 & 194 & $<0.05$ \\
\hline Rank CHX 3 e CHX 10 & 5 & ns & Rank CHX 14 e PTFE 14 & 248,5 & $<0.05$ \\
\hline Rank CHX 3 e CHX 14 & 18,5 & ns & Rank CHX 14 e PTFE 21 & 306,5 & $<0.05$ \\
\hline Rank CHX 3 e CHX 21 & 27 & ns & Rank CHX 21 e PTFE 1 & 154 & $<0.05$ \\
\hline Rank CHX 3 e PTFE 1 & 181 & $<0.05$ & Rank CHX 21 e PTFE 3 & 160,5 & $<0.05$ \\
\hline Rank CHX 3 e PTFE 3 & 187,5 & $<0.05$ & Rank CHX 21 e PTFE 5 & 154 & $<0.05$ \\
\hline Rank CHX 3 e PTFE 5 & 181 & $<0.05$ & Rank CHX 21 e PTFE 7 & 161 & $<0.05$ \\
\hline Rank CHX 3 e PTFE 7 & 188 & $<0.05$ & Rank CHX 21 e PTFE 10 & 185,5 & $<0.05$ \\
\hline Rank CHX 3 e PTFE 10 & 212,5 & $<0.05$ & Rank CHX 21 e PTFE 14 & 240 & $<0.05$ \\
\hline Rank CHX 3 e PTFE 14 & 267 & $<0.05$ & Rank CHX 21 e PTFE 21 & 298 & $<0.05$ \\
\hline Rank CHX 3 e PTFE 21 & 325 & $<0.05$ & Rank PTFE 1 e PTFE 3 & 6,5 & ns \\
\hline Rank CHX 5 e $\mathrm{CHX} 7$ & 1 & ns & Rank PTFE 1 e PTFE 5 & 0 & ns \\
\hline Rank CHX 5 e CHX 10 & 4,5 & ns & Rank PTFE 1 e PTFE 7 & 7 & ns \\
\hline Rank CHX 5 e CHX 14 & 18 & ns & Rank PTFE 1 e PTFE 10 & 31,5 & ns \\
\hline Rank CHX 5 e CHX 21 & 26,5 & ns & Rank PTFE 1 e PTFE 14 & 86 & ns \\
\hline Rank CHX 5 e PTFE 1 & 180,5 & $<0.05$ & Rank PTFE 1 e PTFE 21 & 144 & $<0.05$ \\
\hline Rank CHX 5 e PTFE 3 & 187 & $<0.05$ & Rank PTFE 3 e PTFE 5 & 6,5 & ns \\
\hline Rank CHX 5 e PTFE 5 & 180,5 & $<0.05$ & Rank PTFE 3 e PTFE 7 & 0,5 & ns \\
\hline Rank CHX 5 e PTFE 7 & 187,5 & $<0.05$ & Rank PTFE 3 e PTFE 10 & 25 & ns \\
\hline Rank CHX 5 e PTFE 10 & 212 & $<0.05$ & Rank PTFE 3 e PTFE 14 & 79,5 & ns \\
\hline Rank CHX 5 e PTFE 14 & 266,5 & $<0.05$ & Rank PTFE 3 e PTFE 21 & 137,5 & $<0.05$ \\
\hline Rank CHX 5 e PTFE 21 & 324,5 & $<0.05$ & Rank PTFE 5 e PTFE 7 & 7 & ns \\
\hline Rank $\mathrm{CHX} 7$ e $\mathrm{CHX} 10$ & 3,5 & ns & Rank PTFE 5 e PTFE 10 & 31,5 & ns \\
\hline Rank CHX 7 e CHX 14 & 17 & ns & Rank PTFE 5 e PTFE 14 & 86 & ns \\
\hline Rank CHX 7 e CHX 21 & 25,5 & ns & Rank PTFE 5 e PTFE 21 & 144 & $<0.05$ \\
\hline Rank CHX 7 e PTFE 1 & 179,5 & $<0.05$ & Rank PTFE 7 e PTFE 10 & 24,5 & ns \\
\hline Rank CHX 7 e PTFE 3 & 186 & $<0.05$ & Rank PTFE 7 e PTFE 14 & 79 & ns \\
\hline Rank CHX 7 e PTFE 5 & 179,5 & $<0.05$ & Rank PTFE 7 e PTFE 21 & 137 & $<0.05$ \\
\hline Rank CHX 7 e PTFE 7 & 186,5 & $<0.05$ & Rank PTFE 10 e PTFE 14 & 54,5 & ns \\
\hline Rank CHX 7 e PTFE 10 & 211 & $<0.05$ & Rank PTFE 10 e PTFE 21 & 112,5 & ns \\
\hline Rank CHX 7 e PTFE 14 & 265,5 & $<0.05$ & Rank PTFE 14 e PTFE 21 & 58 & ns \\
\hline Rank CHX 7 e PTFE 21 & 323,5 & $<0.05$ & & & \\
\hline
\end{tabular}


adjunctive use of CHX chip is a clinically safe and effective treatment option for long-term management of chronic periodontitis. CHX chip, when used as an adjunct to SRP, significantly reduces loss of alveolar bone, improve the clinical parameters and modify the subgingival flora $(3,5,18)$.

Otherwise, Carvalho et al. (8) failed to observe any adjunctive effect on subgingival placement of $\mathrm{CHX}$ chips after SRP. Grisi et al. (9) concluded that CHX chip did not provide any clinical and microbiological bene-fit beyond that achieved with conventional SRP, after a 9-month period. CHX chip treatment of adult periodontitis lesions provides little or no additional antimicrobial benefits in comparison to thorough SRP alone (10). In a review study, Cosny et al. (4) concluded that the clinical and microbiological data currently available on the CHX chip are limited and conflicting and more research are needed to elucidate the additional value of the CHX chip when uses as an adjunct to scaling and rooting planning.

CHX chip consists in a degradable matrix of crosslinked hydrolyzed gelatin (18). According to the present study, the CHX chip degradation process persisted until the 21-day period. Comparing to the control group, CHX chip presents a strong inflammatory reaction that can put in check the biocompatibility of the material. The CHX chip could be an important additional item in the treatment of periodontal disease that not responds well to the traditional treatment. However, unfortunately, with the actually results about the CHX chip, the real benefit is not sure, and more studies are needed to evaluate the cytotoxic effect of $\mathrm{CHX}$, and the biocompatibility of CHX chip.

\section{References}

References with links to Crossref - DOI

1. Slots J. The search for effective, safe and affordable periodontal therapy. Periodontol 2000. 2002;28:9-11.

2. Walker CB, Karpinia K, Baehni P. Chemotherapeutics: antibiotics and other antimicrobials. Periodontol 2000. 2004;36:146-65.

3. Paolantonio M, D’Angelo M, Grassi RF, Perinetti G, Piccolomini R, Pizzo G, et al. Clinical and microbiologic effects of subgingival controlled-release delivery of chlorhexidine chip in the treatment of periodontitis: a multicenter study. J Periodontol. 2008;79:271-82.

4. Cosyn J, Wyn I. A systematic review on the effects of the chlorhexidine chip when used as an adjunct to scaling and root planing in the treatment of chronic periodontitis. J Periodontol. 2006;77:25764.

5. Mizrak T, Güncü GN, Caglayan F, Balci TA, Aktar GS, Ipek F. Effect of a controlled-release chlorhexidine chip on clinical and microbiological parameters and prostaglandin E2 levels in gingival crevicular fluid. J Periodontol. 2006;77:437-43.

6. Soskolne WA, Proskin HM, Stabholz A. Probing depth changes following 2 years of periodontal maintenance therapy including adjunctive controlled release of chlorhexidine. J Periodontol. 2003;74:420-7.

7. Azmak N, Atilla G, Luoto H, Sorsa T. The effect of subgingival controlled-release delivery of chlorhexidine chip on clinical parameters and matrix metalloproteinase- 8 levels in gingival crevicular fluid. J Periodontol. 2002;73:608-15.

8. Carvalho J, Novak MJ, Mota LF. Evaluation of the effect of subgin- gival placement of chlorhexidine chips as an adjunct to scaling and root planing. J Periodontol. 2007;78:997-1001.

9. Grisi DC, Salvador SL, Figueiredo LC, Souza SL, Novaes AB, Grisi MF. Effect of a controlled-release chlorhexidine chip on clinical and microbiological parameters of periodontal syndrome. J Clin Periodontol. 2002;29:875-81.

10. Daneshmand N, Jorgensen MG, Nowzari H, Morrison JL, Slots J. Initial effect of controlled release chlorhexidine on subgingival microorganisms. J Periodontal Res. 2002;37:375-9.

11. Houri-Haddad Y, Halabi A, Soskolne WA. Inflammatory response to chlorhexidine, minocycline $\mathrm{HCl}$ and doxycycline $\mathrm{HCl}$ in an in vivo mouse model. J Clin Periodontol. 2008;35:783-8.

12. Chang YC, Huang FM, Tai KW, Chou MY. The effect of sodium hypochlorite and chlorhexidine on cultured human periodontal ligament cells. Oral Surg Oral Med Oral Pathol Oral Radiol Endod. 2001;92:446-50.

13. Faria G, Cardoso CR, Larson RE, Silva JS, Rossi MA. Chlorhexidine-induced apoptosis or necrosis in L929 fibroblasts: A role for endoplasmic reticulum stress. Toxicol Appl Pharmacol. 2009;234:25665.

14. Onçağ $\mathrm{O}$, Hoşgör $\mathrm{M}$, Hilmioğlu $\mathrm{S}$, Zekioğlu $\mathrm{O}$, Eronat $\mathrm{C}$, Burhanoğlu D. Comparison of antibacterial and toxic effects of various root canal irrigants. Int Endod J. 2003;36:423-32.

15. Faria G, Celes MR, De Rossi A, Silva LA, Silva JS, Rossi MA. Evaluation of chlorhexidine toxicity injected in the paw of mice and added to cultured 1929 fibroblasts. J Endod. 2007;33:715-22.

16. Mariotti AJ, Rumpf DA. Chlorhexidine-induced changes to human gingival fibroblast collagen and non-collagen protein production. J Periodontol. 1999;70:1443-8.

17. Almazin SM, Dziak R, Andreana S, Ciancio SG. The effect of doxycycline hyclate, chlorhexidine gluconate, and minocycline hydrochloride on osteoblastic proliferation and differentiation in vitro. J Periodontol. 2009;80:999-1005.

18. Jeffcoat MK, Palcanis KG, Weatherford TW, Reese M, Geurs $\mathrm{NC}$, Flashner M. Use of a biodegradable chlorhexidine chip in the treatment of adult periodontitis: clinical and radiographic findings. J Periodontol. 2000;71:256-62.

19. Monteiro AS, Macedo LG, Macedo NL, Balducci I. Polyurethane and PTFE membranes for guided bone regeneration: histopathological and ultrastructural evaluation. Med Oral Patol Oral Cir Bucal. 2010;15:e401-6.

20. De Macedo NL, De Macedo LG, Monteiro Ado S. Calcium sulfate and PTFE nonporous barrier for regeneration of experimental bone defects. Med Oral Patol Oral Cir Bucal. 2008;13:E375-9.

21. Chang YC, Yang SF, Lai CC, Liu JY, Hsieh YS. Regulation of matrix metalloproteinase production by cytokines, pharmacological agents and periodontal pathogens in human periodontal ligament fibroblast cultures. J Periodontal Res. 2002;37:196-203.

22. Giannelli M, Chellini F, Margheri M, Tonelli P, Tani A. Effect of chlorhexidine digluconate on different cell types: a molecular and ultrastructural investigation. Toxicol In Vitro. 2008;22:308-17. 